\title{
A Brand Premium Pricing Model for Digital Music Market
}

\author{
Tobey H. Ko and Henry Y. K. Lau
}

\begin{abstract}
This paper proposes an alternative pricing scheme for the digital music market, where the musician's differentiated characteristics such as fame, popularity, and listener share - in the form of consumer's brand premium multiplier - is incorporated into the pricing decision by the record label. We consider consumers are differentiated by two types of characteristics: the brand premium multiplier towards the musician, and the general preference to music. We argue that in the co-existence of free music streaming, the retail price of a musical product should be allowed to vary in a manner that reflects the differences in market environment and the musician's characteristics. The results of the proposed model provide a toolkit for the recording industry to effectively price its products in a market that is constantly evolving.
\end{abstract}

Index Terms-Brand premium, music streaming, pricing, self-selection.

\section{INTRODUCTION}

The music industry has been continuously exploring and creating alternative business models to develop new sources of stable income stream and dedicated global audience. The music streaming business model, lead by global brands like Spotify and Deezer, has gained increasing popularity in recent years, offering huge diversity to the industry's mix of revenue streams. However, even as many reports [1], [2] praising the tremendous potential of the online streaming business model and regard them as the future for the music industry, the study by Generator Research [3] revealed the financial aspect of music streaming is a disastrously unprofitable one; where selling one song on the iTunes Music store can be hundred times more profitable than one streaming play on the Spotify platform.

As compelling as the potential size of listener these online music streaming platform could bring to the record labels and musicians, the profitability side of the business model is perhaps less promising or even frightening to the industry. The online music streaming business model can easily be the greatest disruption to the music industry since the rise and fall of Naspter in late 1990s and early 2000s, yet it is evident this new way of discovery and consumption of music is still at its infancy and not fully compatible with the traditional music transaction models the industry is used to have, and such incompatibility is likely to lead to loss of revenue stream to

Manuscript received December 24, 2014; revised February 28, 2015

The authors are with the Department of Industrial and Manufacturing Systems Engineering, the University of Hong Kong, Pokfulam, Hong Kong (e-mail: magicfor@hku.hk, hyklau@hku.hk). the producers and investor in the industry. One recent alarming case was Taylor Swift's removal of her entire music catalog from the popular music streaming platform Spotify, stating "valuable things should be paid for. It's my opinion that music should not be free..." [4]. The incident echoed strongly with the on-going debate of the appropriateness of the free-to-listen business model of music streaming, as to how much should the producers be compensated to provide them incentive to continue with this way of publishing their music.

Given the public goods nature of the music as a piece of product, making the product always available on the cloud and free to access would indeed lead to loss in revenue from the sale of the same good in both physical and digital medium. On the other hand, the online music streaming platform may also act as a major promotional channel and primary source of income for lesser known musicians who are perhaps unable to sell their music in other retailing platforms. Having witnessed consumers shifting back to pirating the Taylor Swift music album illegally after the removal of the streaming catalog, suggesting banning of music streaming is perhaps less effective as a way to persuade consumers into purchasing the musical product, thus the need to explore alternative ways to attract more music listeners into purchasing the product with the co-existence of free music streaming service is imminent In this paper, we propose a novel self-selection model incorporating the fame of the musician into the pricing decision of the musical product, as opposed to the level pricing scheme that is widely used in practice, we hypothesize this more flexible pricing scheme can benefit record labels of different sizes.

Given that music is a form of information goods where the fixed cost of production is extremely high with negligible variable cost of production, the traditional fixed cost pricing approach cannot be applied here. We looked deep into the literature discussing the self-selection pricing decision of information goods in the existence of an alternative product. Among those literature, the proposal to practice second-degree price discrimination and bundling are most favored by researchers in this field.

The second-degree price discrimination pricing scheme is favored by a large number of researchers in which the producer releases the information goods in more than one quality, and vertically differentiate the products to sell to heterogeneous consumers. The use of vertical differentiation in pricing information goods was first proposed by Mussa and Rosen [5] in which the monopolist produces a line of product of different quality in the same generic type, where the copy is a low-quality substitute of the original; the heterogeneous consumers engages in a process of self-selection and purchases the product to maximize their utility. Although we still observe a few record labels continue to release an 
additional deluxe version of the music album to price discriminate the high valuation consumers, this practice is not supported by more recent studies. Several researchers have tried to further develop the model of vertical differentiation pricing scheme on seller of information goods. Meyer [6], Jones and Mendelson [7] has examined a market of information goods with heterogeneous consumers, their model finds that price discrimination is not optimal to the monopolist when its marginal cost is set to zero. Variation of the vertical differentiation is the information goods pricing model by Belleflamme [8], where he analyzed the effect of copying in a market for information goods with 1 monopolist in both short-run and long-run; the result shows price discrimination is not optimal when copies are not a low-quality alternative to the originals. Findings from these papers show vertical price discrimination alone is not an optimal pricing scheme for digitized information goods as the copies are near-perfect substitutes of the originals.

Another pricing scheme proposed by researchers is the use of bundling - selling a large number of differentiated goods for a fixed price so the company does not need to identify and differentiate heterogeneous consumers with different preferences. The benefit of bundling goods was first proposed by Stigler [9] using a model with two goods of negatively related consumer valuation, the model has shown that the monopolist's profit can be improved by bundling the two goods and sell at a fixed price. The bundling approach is currently widely used in the market for information goods, for instance bundling ten songs in a music album is already a bundling of differentiated songs. In the context of digital information goods, Bakos and Brynjolfsson [10] was the first to discuss bundling of a large number of information goods on the internet. Their results suggest that offering a menu of different bundles of large collection of information goods makes the consumer's valuation fairly predictable, thus a multi-product monopolist can achieve a higher profit by implementing the bundling strategy. A customized bundling strategy was introduced by Hitt and Chen [11] which allows the consumer to choose up to a certain number of goods from a pool of digital goods, their model show such bundling strategy is particularly attractive when the monopolist is selling information goods of high value and the heterogeneous consumers are budget constrained. An empirical study of the music CD bundling strategy by Lee, Tsai, and $\mathrm{Wu}$ [12] suggested that successful bundling directs the consumer's attention away from the pricing attribute to the content of the product, so a mixed-bundling strategy is more effective in influencing consumer's purchasing intent.

Apart from the conventional pricing schemes, various alternative pricing schemes are proposed by the researchers. Sundararajan [13] analyzed the trade-off between transaction cost and usage of the information good, and concluded that in the presence of any positive transaction costs, sells of information good should offer their customers a combination of usage-based pricing (unit pricing) and unlimited-usage fixed-fee pricing (product bundling). The cooperative pricing scheme proposed by Buxmann, Strube, and Pohl [14] was an attempt to integrate different functions in the digital music supply chain in order to increase the turnover from music downloads. The paper proposed a model to find the profit maximizing prices for digital music, and provided a mechanism to split up the revenue between digital music supply chain partners. A more recent literature by Kubiszewski, Farley, and Costanza [15] discussed about the public good property of information which is enhanced with increased use; a common ownership of information is suggested to increase information transfer and produce a greater rate of innovation, in this sense, information should be obtained free of charge.

Only very few studies have incorporated the concept of brand premium into their pricing models. The theoretical model constructed by Gallaugher and Wang [16] identified the brand premium in the software market in the form of consumer mind-share, and such mind-share is directly related to the software company's market share and profitability. Wang and Zhang [17] examined the effect of free sampling in a monopolistic market with both optimistic consumer and pessimistic consumer; their findings show the monopolist can benefit from the free sampling process under some market conditions. Results of these papers give support to the need of keeping the online music sampling platform, as these platforms provide a way for the record labels to assess and improve the brand premium of the musicians.

In this paper, we draw inspiration from the literature in the brand premium and second-degree price discrimination field, and propose a novel self-selection model utilizing the musician's popularity and fame in the market at the time of releasing the musical product as a way to better price the product to maximize the record label's profitability from the sale of the product. With the inclusion of musician's popularity and brand premium in the price, the record label is able to adjust the selling price of their product base on the observation to the market's reception to the musician and the musical product itself. As a result, musicians and music that are well received by the market and those that are less well received would be priced in a well differentiated manner to maximize the record label's profit.

The rest of the paper is organized into four sections: Section II presents the basic setting of our proposed model. Section III provides general discussion on the behavior of the proposed model. Section IV gives a numerical example on the expected results of the proposed model. Section $\mathrm{V}$ presents a short conclusion and provides direction on future research into the topic.

\section{MODEL DESCRIPTION}

We consider a market in which a profit-maximizing record label selling a piece of product in the consumer market called digital music. The production and promotion of the digital music is financed by the record label, and the production process is done by a third-party producer signed on by the record label known as the musician. In such case, the musical product produced by the musician is a monopolistic product, where the music not only carries a quality trait that is directly related to the production cost of the music, it also features the unique characteristics of the musician. Given the uniqueness of the musician, the consumers' likeliness of the artist are also be taken into consideration when he/she is making the 
consumption decision.

\section{A. Musician}

The musician in the proposed model does not interact directly with the consumers. For instance, the musicians must rely on the investment from the record label to produce and promote musical product that are then sold to the consumers. In other words, the record label is acting as an intermediary between the musician and the consumers.

Although the musicians do not interact with the consumers, the fame and popularity of the musician have direct effect on the brand premium based pricing model that we are proposing In the context of the model, the musicians differentiate with one another in three dimensions: fame $(\mu \geq 1)$, popularity $(\sigma \geq$ $0)$, and listener share $(\gamma \sim U(0,1))$, where the fame $(\mu)$ and popularity $(\sigma)$ popularity of the musician together forms the distribution of the musician's brand premium $\beta \sim N\left(\mu, \sigma^{2}\right)$ among the musician's share of listeners $\gamma$. To briefly explain the composition of the musician's characteristics, we consider a market of potential consumers of size $G$, where $N$ out of the $G$ consumers are aware of the musician and thus have an understanding on the musician to develop a brand premium in purchasing the musician's musical product. The musician's listener share $\gamma$ is thus equal to $N / G$, corresponding to the proportion of consumers the musician has in the potential consumer market. A brand premium multiplier only applies to the listeners within the size $N$ population, with the individual brand premium multiplier of each consumer falls into the distribution of the musician's brand premium $\beta$. For the composition of the musician's brand premium $\beta$, we consider the fame $\mu$ as a reflection of the historical achievement of the musician; for instance, musicians who have won awards and have high charting songs in the past would score a higher value on his/her fame, and those who hardly have any achievements in the past would score a fame value close to 1 . The popularity $\sigma$ dimension of the brand premium $\beta$ relates to the general perception on the musician's brand value from the consumers; the popularity $\sigma$ would be small if the listeners have a more unified perception on the musician, whereas $\sigma$ would be large if the listeners' opinion on the musician is more diversified. Overall, the model would consider both the musician's brand premium $\beta$ (as a combination of fame and popularity) and the listener share $\gamma$ when the record label is making the profit-maximizing pricing decision.

\section{B. Consumers}

Recall from the description in the musician section, the market for the musical product is observed by listeners in the market of mass $N$. The size of the consumer market $N$ reflects the number of music listeners who would potentially make a unit purchase on the specific digital music produced by the musician. The consumers are assumed to be utility-maximizing and are heterogeneous in two dimensions: the consumer's general preference to music $\theta$, and the consumer's brand premium multiplier $\beta$ towards the musician. For an individual consumer $i$ in the market, $\beta^{i}=Z^{i} \sigma+\mu$ for $i \forall$ $N$, where $Z \sim N(0,1)$ is the consumer's musician specific (brand) preference. Constructing the consumer's brand premium multiplier in this way allows us to further the analysis on the model behavior, and gain more understanding of the variation of musician's different qualities on the consumers' consumption and the record label's pricing decision. The consumer's general preference to music $\theta$ is assumed to be uniformly distributed $\theta \sim U[0,1]$, where consumers having a higher musical preference $\theta$ will consume more music in general relative to those having a lower $\theta$. When a piece of digital music is released in the market, the consumers have two ways to consume the digital music: purchase the digital music at price $p$, or stream the music free-of-charge from online music streaming platform at a constant opportunity cost $(1-\gamma) K$, where $\mathrm{K}$ is an opportunity cost constant. We assume the consumers' opportunity cost for music streaming is negatively related to the listener share $\gamma$ of the musician in honor of the existence of a potential positive network effect, where the consumer may derive more utility from streaming the song if the musician has more listeners at the moment, consistent with the bandwagon effect.

$$
U^{\theta}= \begin{cases}\beta^{\theta} \theta q-p, & \text { Purchase } \\ \theta q-(1-\gamma) K, & \text { Free Streaming } \\ 0, & \text { Otherwise }\end{cases}
$$

where,

$$
\beta^{\theta}=Z^{\theta} \sigma+\mu
$$

s.t.

$$
p>(1-\gamma) K
$$

Equation (1) denotes the utility derived from consuming the given musical product for a consumer with general music preference $\theta$. The consumer's utility function is constructed as a self-selection model, where the consumer chooses among purchasing, streaming or not consuming the musical product at all to maximize his/her utility. From (1), it is obvious the consumer's decision to purchase or stream the digital music is independent of the quality of music $q$, where $0<q<1$, but rather the brand premium multiplier $\beta$ the consumer has on the music artist. By restricting the price of digital music $p$ to be always greater than the opportunity cost of free streaming (1$\gamma) K$, the consumers' utility function guarantees only those who truly appreciate the music artist would purchase the digital music (as opposed to purchase the musical product because it is priced too low). The decision to stream the music free-of-charge or not consume the music once-and-for-all is, however, highly relevant to the quality of digital music.

\section{Record Label}

We assume the record label makes money from the digital music out of two different revenue streams: sale of digital music through digital music retailing platform, and music streaming revenue through music streaming platform. At this point we assume the music album has already been produced, thus the record label has already incurred a fixed cost of production $F(q)$ to produce the music album; in other words, quality of music album has already been determined at this pricing stage. To reflect the effect of quality to the demand for 
music streaming, we further assume the fixed cost function $F(q)$ is a strictly increasing convex function with $F^{\prime}(q)>0$ and $F^{\prime} '(q)>0$. By assuming the convexity of the fixed cost function, it implies that it becomes increasingly costly to produce higher quality digital music. For simplicity, we assume the function for fixed cost of production to be $F(q)=$ $\mathrm{C} q^{2} / \mu$, where $C$ is a product cost constant, and a positive relation to the fame (historical achievement) of the musician is assumed to reflect his/her cost in making music; the higher the music artist's fame $(\mu)$, the lower the cost to product music of a given quality $(q)$.

$$
\operatorname{aug} \max _{(p)} \pi=(1-\alpha) p D(p)+\bar{p} S(q)-\frac{C q^{2}}{\mu}
$$

The record label's decision in the model is to choose a price $p$ to maximize the profit $\pi$ derived from sales $D(p)$ and streaming $S(q)$ of the musical product. With this in mind, the objective function for the profit-maximizing record label is constructed as shown in (4), where $\alpha$ denotes the constant share of sales revenue demanded by the music retail platform as commission, $\bar{p}$ is the fixed amount of revenue from each streaming play of the musical product. Given that the record label's revenue derived from each music streaming is pre-determined by the music streaming platform, only the price of digital music $p$ is relevant to the record label's profit-maximizing decision at this stage.

\section{MODEL DISCUSSION}

We discuss the important properties of the proposed model in this section. Specifically, the dynamics between different variables in the proposed model will be discussed to provide a general decision support framework to the record label as to how to react to variations in the market dynamics to achieve desirable effects.

\section{A. Critical Values}

We derive critical values on the consumers' characteristics based on the consumer's utility function shown in (1). In our analysis, we analyze the consumer's musician specific (brand) preference $Z$ instead of the brand premium multiplier $\beta$, as $\beta$ relies heavily on the different qualities of the musician.

\section{1) Marginal consumer on demand for purchase}

Our analysis considers the case where a marginal consumer is indifferent to making a purchase of the musical product and streaming the music from online music streaming platforms. In such case, a marginal consumer would satisfy the condition in (5):

$$
\beta \theta q-p=\theta q-(1-\gamma) K
$$

Analyzing the marginal consumer condition in (5) yields the critical values

$$
\theta_{P}^{*}=\frac{p-(1-\gamma) K}{(Z \sigma+\mu-1) q}
$$

and

$$
Z_{P}^{*}=\frac{p-(1-\gamma) K+(1-\mu) \theta q}{\sigma \theta q}
$$

where $\theta_{P}^{*}$ denotes the marginal value of general music preference to purchase for consumers in every given level of musician specific (brand) preference $Z$, and $Z^{*}{ }_{P}$ denotes the marginal value of musician specific (brand) preference to purchase for consumers in every given level of general music preference $\theta$.

The importance of the critical values give raise the proportion of consumers at each level of $Z$ and $\theta$ who would choose purchase over streaming of the musical product. For instance, for each level of $Z$, all the consumers with $\theta$ higher than the marginal value of general music preference $\theta^{*}{ }_{P}$ would purchase the musical product; where for each level of $\theta$, all the consumers with $Z$ higher than the marginal value of musician specific (brand) preference $Z^{*}$ would choose purchase over streaming. These values can later be used to derive the overall demand for purchase.

\section{2) Marginal consumer on demand for streaming}

Using the same rationale, we analyze the case where a marginal consumer is indifferent to streaming the music from online music streaming platform and not consume the music once-and-for-all. In such case, a marginal consumer would satisfy the condition in (8):

$$
\theta q-(1-\gamma) K=0
$$

Analyzing the marginal consumer condition in (8) yields the critical value:

$$
\theta_{S}^{*}=\frac{(1-\gamma) K}{q}
$$

where the $\theta^{*}$ denotes the marginal value of general music preference to streaming for consumers in every given level of musician specific (brand) preference $Z$. As it is shown in (9), the critical level $\theta^{*}$ is independent of the level of $Z$, but instead negatively related to the musician's listener share $\gamma$ and the quality of the musical product $q$. In such case, all consumers with $\theta$ higher than the marginal value of general music preference $\theta^{*}$ would stream the musical product on from the online music streaming platforms, and do not consume at all if otherwise.

\section{3) Equilibrium demand for purchase and streaming}

The equilibrium demand for purchase $D(p)$ and streaming $S(q)$ can be derived by further analyzing the critical values obtained above. Fig. 1 is drawn to illustrate the equilibrium market demand for purchase, free streaming, and no consumption, where the region for purchasing is shaded with vertical strip pattern, the region for free streaming is shaded with gray. As a result, consumers with general preference to music $\theta$ and musician specific (brand) preference $Z$ falling into these regions shaded in Fig. 1 would choose to perform the respective utility maximizing action of that region.

The curve drew in bold in Fig. 1 represents the characteristics of the marginal consumers. More specifically, there are 3 critical values labeled in the figure that are of high 
importance: $Z^{*}{ }_{1}$ denotes the lowest possible value of musician specific (brand) preference $Z$ that a consumer of any general preference to music $\theta$ would consider making a purchase for a given piece of musical product. The $\theta_{\mathrm{S}}^{*}$, as discussed above, denotes the marginal value of general music preference to streaming, and it represents the lowest possible value of general preference to music $\theta$ that a consumer of any musician specific (brand) preference $Z$ would consider to stream the music from online music streaming platforms. Finally, $Z^{*}$ denotes the musician specific (brand) preference of a consumer, when coupled with a general preference to music of $\theta_{S}^{*}$, would be indifferent to purchase, streaming, and not consuming the piece of musical product at all. We provide a brief derivation on these critical values below.

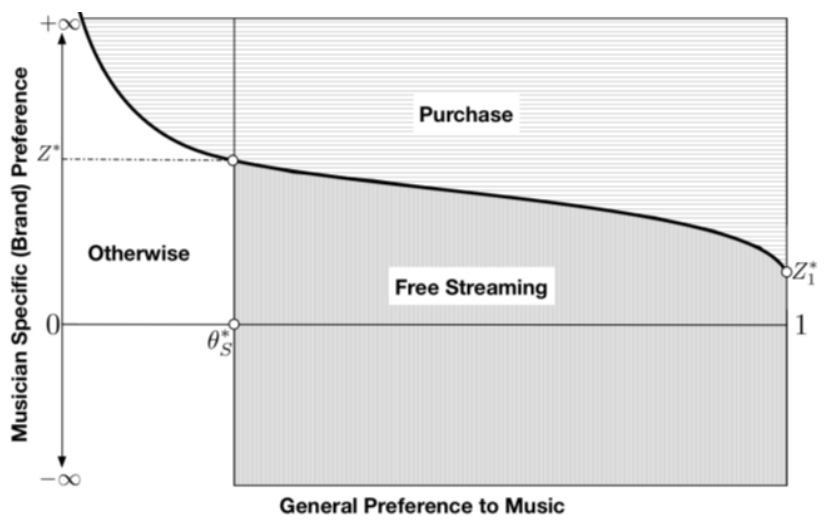

Fig. 1. Equilibrium demand for purchase and streaming.

Since the general preference to music $\theta$ is bounded between 0 and 1 , the critical value $Z^{*}$ can be derived by substituting $\theta$ $=1$ into (7) to obtain the lowest value of musician specific (brand) preference $Z$. The composition of $Z_{1}^{*}$ is shown in (10):

$$
Z_{1}^{*}=\frac{p-(1-\gamma) K+(1-\mu) q}{\sigma q}
$$

Using the same rationale as above, the position of $Z^{*}$ can be determined by substituting the value of $\theta^{*}{ }_{S}$ in (9) into that of $Z_{P}^{*}$ in (7). The composition of $Z^{*}$ is shown in (11):

$$
Z^{*}=\frac{p-\mu(1-\gamma) K}{\sigma(1-\gamma) K}
$$

For a given market price $p$, we further assume there exist a critical value for musician's popularity diversity $\sigma$, and listener share $\gamma$ in terms of $\hat{\sigma}$ and $\hat{\gamma}$, such that,

$$
\frac{\partial \pi}{\partial \sigma}=0 \text { and } \frac{\partial^{2} \pi}{\partial \sigma^{2}}<0
$$

and

$$
\frac{\partial \pi}{\partial \gamma}=0 \text { and } \frac{\partial^{2} \pi}{\partial \gamma^{2}}<0
$$

The critical values of the musician's characteristics $\hat{\sigma}$ and $\hat{\gamma}$ can be interpreted as the ideal level of popularity diversity and listener share under a given price $p$ for any musicians.

A number of observations can be deduced from the demand curves for purchase and streaming in Fig. 1. Through the understanding of these demand characteristics, a record label can make optimal decisions accordingly based on their observed market dynamics in the music industry.

Proposition 1: A higher musician's fame $\mu$ would drive up both the price $p$ and the record label's profit $\pi$, and vice versa.

Proof: Based on Fig. 1, the demand for purchase is negatively related to the position of $Z^{*}$, where the higher the position of $Z^{*}$ the less the demand for purchase. It is also shown in (10) the level of $Z^{*}{ }_{1}$ is negatively related to the level of $\mu$, so a higher fame is represented by a downward shift of the curve for marginal consumers, indicating a higher demand for purchase. Since a higher fame means the consumers generally has a higher brand premium multiplier which increase their willingness to pay, it is natural to raise price and shift up the marginal consumer curve to capture this higher willingness to pay. Even if the price increase to an extent that the level of $Z_{1}^{*}$ is back to its original position, the record label still enjoys a higher profit from the increased selling price. As the musician's fame is irrelevant to the demand for streaming, we can interpret the higher sales as an improvement to the profitability.

Following the claims in Proposition 1, it is always more preferable for the record label to sell musical products that are produced by musicians that are more acclaimed, because the record label would be able to charge more on these musical products and enjoy a higher profit from it.

Proposition 2: For musicians who are differentiable in any of his/her musician characteristics, the record label should seek to price their musical products differently to maximize expected profit.

Proof: As shown in proposition 1, the record label should always price higher for musicians with higher fame. For a given price $p$, the critical values $\hat{\sigma}$ and $\hat{\gamma}$ in (12), and (13) represent the efficient levels of popularity diversity and listener share under that pricing point. As a result, a deviation from the optimal values reflect an inefficiency that can be captured by charging the profit maximizing price at the given levels of musician characteristics.

Suppose the record label cooperates with a portfolio of musicians in releasing their musical products, the record label would have an incentive to only sign with well-known musicians with an established listener base if the prices were the same for all musical products. Differentiated pricing allows the record labels to diversify their portfolio, not only would there be more musical products available in the market, pricing competitively would also maximize the profit for the record label.

Proposition 3: A more diversified popularity $\sigma$ has ambiguous effects on the profit-maximizing pricing decision and the demand for purchase.

Proof: Refer to (6), the demand for purchase $(d(p))$ for consumers having musician specific (brand) preference $i$, for $i$ $\forall Z$, can be written as

$$
d(p)=\frac{p-(1-\gamma) K}{(Z \sigma+\mu-1) q}
$$


By the same token, the demand for purchase $D(p)$ for all consumers as a whole is

$$
\begin{aligned}
& \int_{Z_{1}^{*}}^{+\infty} d(p) d Z \cdot G(Z) \\
& =\left[\frac{(p-K(1-\gamma)) \ln (\sigma Z+\mu-1)}{q \sigma}\right]_{Z_{1}^{*}}^{+\infty} \cdot G(Z)
\end{aligned}
$$

where $G(Z)$ is the distribution function of $Z$.

We can derive from (14) that $d(p)$ is positively related to the musician's popularity diversity, yet from (15) its effects to $D(p)$ is ambiguous as it is positively related to $d(p)$ but negatively related to $Z^{*}$ as shown in (10).

The decision in pricing and its effect to demand for purchase depend on the position of the musician's original popularity diversity. By comparing it to the critical value of musician's popularity $\hat{\sigma}$ in (12), the record label should seek to reduce price at times of an increased popularity diversity to improve demand for purchase if $\sigma$ is smaller than $\hat{\sigma}$, and vice versa.

Proposition 3 draw implication to pricing arrangements of the musical products based on the genre (or market trend) of these products. The musician's popularity diversity $\sigma$ can be interpreted as a proxy to the genre of music that is more or less generally accepted in the market, such that a more popular genre would have a smaller $\sigma$ and a less popular genre would have a larger $\sigma$. In this sense, not only should the prices of musical products across different genres be differentiated with each other, the profit maximizing record label should also seek to reduce price when a musical product is in a genre that's slowly fading out of trend.

Proposition 4: The record label derives more revenue from streaming if a musician has a greater listener share $\gamma$, and vice versa. While the effects to the profit-maximizing pricing decision and the demand for purchase is ambiguous.

Proof: Based on (9), $\theta^{*}{ }_{S}$ is negatively related to the musician's listener share $\gamma$. Thus, a higher $\gamma$ would induce a higher demand for streaming as shown in Fig. 1, and there's a higher profit made from the streaming platforms.

Using the same rationale in the proof of proposition 3, the decision in pricing and its effect to demand for purchase depend on the position of the musician's original listener share. If the original listener share is lower than the critical value of musician's listener share in (13), i.e. $\gamma<\hat{\gamma}$, the record label should seek to decrease price at times of an increased listener share to improve demand for purchase, and vice versa.

Our result in proposition 4 provides a general direction to the record labels in making pricing decisions to the musical products that has gain more or losing some listeners. For instance, musicians with relatively little listeners can charge a higher price for their musical products, but the price should be adjusted down when more listeners gain access to their music; on the other hand, popular musicians with large listener base can sell their products at relatively low price, the record label would better off charging a higher price when these popular musicians gain even more listeners.

\section{NUMERICAL EXAMPLE}

The discussion on the demand characteristics provide a basic toolkit for the record labels in making decisions regarding the pricing of the musical products. Yet, the demand curves in Fig. 1 show only the regions of action where a consumer of certain types would perform under a given circumstances, a specific definition of aggregate demand for purchase and streaming could not be deduced from the curve. This is due to the stochastic nature of the consumer's brand preference, where an analytical solution for the profit maximizing price cannot be derived from the model. Instead, the profit maximizing price can only be calculated through simulation or approximation. In this section, we present a numerical example of the proposed model, where the profit maximizing price is computed using simulation. By varying different variables in the model, we intend to show that the proposed brand premium pricing model can generate a higher profit to the record label.

\section{A. Basic Setting}

We conduct the numerical simulation study using the initial variable settings shown in Table I. These values are chosen for illustrative purpose only, and the magnitudes of them do not carry an empirical implication.

\begin{tabular}{|c|c|c|c|c|c|}
\hline \multicolumn{6}{|c|}{ Simulation Constants } \\
\hline$C$ & $q$ & $\alpha$ & $\bar{p}$ & $K$ & $G$ \\
\hline 20 & 0.8 & 0.1 & 0.0005 & 0.2 & 10000 \\
\hline \multicolumn{6}{|c|}{ Simulation Variables } \\
\hline & & & $N$ & & $\gamma$ \\
\hline & & & 1000 & & 0.1 \\
\hline
\end{tabular}

TABLE I: BASIC SETTING FOR NUMERICAL SIMULATION STUDY

TABLE II: INITIAL RESULT FOR NUMERICAL SIMULATION STUDY

\begin{tabular}{cc}
\hline \hline Variables & Optimal Value \\
\hline$D(p)$ & 263 \\
$S(q)$ & 519 \\
$D(p)$ in $\%$ & $26.3 \%$ \\
$S(q)$ in $\%$ & $51.9 \%$ \\
$p$ & 1.80 \\
$\pi$ & 422 \\
\hline \hline
\end{tabular}

TABLE III: VARIATIONS IN PRICE AND PROFIT WITH MODEL DYNAMICS

\begin{tabular}{lcccccc}
\hline \hline & \multicolumn{7}{c}{ Model Dynamics Settings (S) } \\
\cline { 2 - 7 } Variables & S1 & S2 & S3 & S4 & S5 & S6 \\
\hline$\mu$ & 3 & 3 & 3 & 3 & 6 & 10 \\
$\sigma$ & 5 & 5 & 3 & 8 & 5 & 5 \\
$N$ & 3000 & 5000 & 1000 & 1000 & 1000 & 1000 \\
$\gamma$ & 0.3 & 0.5 & 0.1 & 0.1 & 0.1 & 0.1 \\
\hline \hline Results & & & & & & \\
\hline$D(p)$ & 703 & 1098 & 291 & 182 & 281 & 443 \\
$S(q)$ & 1814 & 3276 & 490 & 598 & 498 & 337 \\
$D(p)$ in $\%$ & $23.4 \%$ & $22.0 \%$ & $29.1 \%$ & $18.2 \%$ & $28.1 \%$ & $44.3 \%$ \\
$S(q)$ in $\%$ & $60.5 \%$ & $65.5 \%$ & $49.0 \%$ & $59.8 \%$ & $49.8 \%$ & $33.7 \%$ \\
$p$ & 2.25 & 2.37 & 1.39 & 3.42 & 3.05 & 3.41 \\
$\pi$ & 1442 & 2343 & 360 & 556 & 769 & 1357 \\
\hline \hline
\end{tabular}

Using the values established in the basic simulation setting in Table I, we performed simulation studies to derive the profit maximizing price for the musical product under such basic setting. The profit maximizing price, along with the corresponding profit and the demands for purchase and streaming for that profit maximizing price are shown in Table 
II.

The initial results Table II would be used as a basis in the study of model dynamics in the next section.

\section{B. Model Dynamics Study}

Table III illustrates the variations in profit maximizing price and profit, along with the demands for purchase and streaming associated with them under 6 different model dynamics settings. To explain a little more on the settings in the model dynamics study, any one of the musician's fame $\mu$, popularity diversity $\sigma$, as well as the listener share $\gamma$ are allowed to vary in different model dynamics settings while holding others constant. The results (optimal values) derived from each settings are used to compare with that of the basic setting, the model dynamics can be draw through these comparisons.

\section{Result Implications}

In Table III, S1 and S2 are used to show the effects of the variations in listener share $\gamma$ to price, profit, and the respective demands for purchase and streaming; whereas S3 and S4 are used to show the effects of the variations in popularity diversity $\sigma$ to price, profit, and the respective demands for purchase and streaming; lastly, S5 and S6 are used to show the effects of the variations in musician's fame $\mu$ to price, profit, and the respective demands for purchase and streaming. By comparing the results in S1 - 6 with the initial result in Table II, we deduct the following conclusions regarding the proposed model:

1) Given the same market for potential consumers $(G)$, a musician with a higher listener share $\gamma$ is able to charge a higher price to maximize the record label's profit. Although the increase in price is accompanied with a decrease in proportion of purchasing consumers and profit derived from each purchasing consumers, more consumers are sharing the large fixed cost of production associated with information goods, thus giving the record label an incentive to sell to more consumers even at a lower profit per purchasing consumer.

2) The record label sees an increase in profit and increase in profit maximizing price at the same time when the musician's popularity diversity $\sigma$ raises. The increase in profit is coupled with a significant decrease in proportion of purchasing consumers, implying the record label should concentrate on selling to the high brand premium multiplier consumers when the musician's popularity is largely diversified.

3) The musician's fame $\mu$ has the most significant impact on the record label's profitability and price charged on the musical product. In the simulation study, the profit maximizing price, the record label's profit, and the demand for purchase all increase when the musician's fame $\mu$ increase from 3 to 6 to 10 . That is to say, the musician's fame $\mu$ can very well serve as an indicator to the record label's profitability when planning the production and release of musical products. Especially when resources are limited, the record label would see a higher profit if it devotes the limited resources on a more well-known musician, rather than one little are known in the market.
The conclusions drawn in the numerical example echo with the propositions in the model discussion. The implications in both model discussion and numerical example can serve as a direction for the record labels to make optimizing decisions, and provide them a toolkit to cope with the dynamics in the music market.

\section{CONCLUSION}

This paper presents a brand premium based pricing model in a market with co-existence of retailing and free streaming of musical product. In our model of self-selection with heterogeneous consumers, we showed that the high level of differentiation among musicians brings forward a need for the record labels to price the musical products in a more flexible manner, based on the consumers' brand premium to be more specific. Our paper contributes to the existing literature on pricing of information goods by introducing the variables in relation to the perception of the consumers into the pricing model. Our result suggests that the consumer perception (brand premium multiplier) plays an important role in the record label's pricing decision, as well as its profitability. To further extend our work, we would continue working on defining the process of forming a brand premium multiplier, which can extend to provide a way for the record labels to act on and influence the consumers.

\section{REFERENCES}

[1] IFPI, "Digital Music Report," International Federation of the Phonographic Industry, London, 2014.

[2] Next Big Sound, 2013: The Year in Rewind, 2013.

[3] Generator Research, Digital Music Subscription Services: 2013, 2013.

[4] T. Swift, For Taylor Swift, the Future of Music Is a Love Story, 2014.

[5] M. Mussa and S. Rosen, "Monopoly and product quality," Journal of Economic Theory, vol. 18, no. 2, pp. 301-317, 1978.

[6] D. W. Meyer, "Essays on quality and product differentiation," University of Michigan, 1996.

[7] R. Jones and H. Mendelson, "Product and price competition for information goods," presented at Ninth Workshop on Information Systems and Economics, 1998.

[8] P. Belleflamme, "Pricing information goods in the presence of copying," The Economics of Copyright: Developments in Research and Analysis, p. 26, 2003.

[9] G. J. Stigler, "United States v. Loew's Inc.: A Note on Block-Booking," The Supreme Court Review, vol. 1963, pp. 152-157, 1963.

[10] Y. Bakos and E. Brynjolfsson, "Bundling and Competition on the Internet," Marketing Science, vol. 19, no. 1, pp. 63-82, 2000.

[11] L. M. Hitt and P. Chen, "Bundling with customer self-selection: A simple approach to bundling low-marginal-cost goods," Management Science, pp. 1481-1493, 2005.

[12] H.-C. Lee, D. C. Tsai, and M.-D. Wu, "The dilemma of mixed bundles: The effects of price, brand, and content preference on music CD bundle choices," Journal of Retailing and Consumer Services, vol. 18, no. 5, pp. 455-462, 2011.

[13] A. Sundararajan, "Nonlinear pricing of information goods," Management Science, vol. 50, no. 12, pp. 1660-1673, 2004.

[14] P. Buxmann, J. Strube, and G. Pohl, "Cooperative pricing in digital value chains - the case of online music," Journal of Electronic Commerce Research, vol. 8, no. 1, pp. 32-40, 2007.

[15] I. Kubiszewski, J. Farley, and R. Costanza, "The production and allocation of information as a good that is enhanced with increased use," Ecological Economics, vol. 69, no. 6, pp. 1344-1354, 2010.

[16] J. M. Gallaugher and Y.-M. Wang, "Understanding network effects in software markets: evidence from web server pricing," MIS Quarterly, pp. 303-327, 2002.

[17] C. A. Wang and X. M. Zhang, "Sampling of information goods," Decision Support Systems, vol. 48, no. 1, pp. 14-22, 2009. 


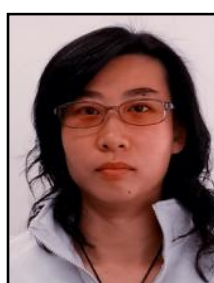

goods.
Tobey H. Ko received the B.Eng degree in industrial engineering and technology management and the B.BA degree in finance from the University of Hong Kong, in 2011 and 2012, respectively. She is currently working towards the Ph.D. degree at the Department of Industrial and Manufacturing Systems Engineering, University of Hong Kong, Hong Kong.

Her research interests include operations research and decision support system for digital information

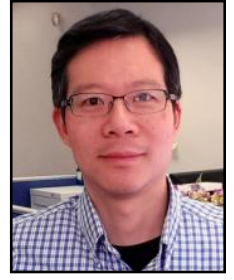

Henry Y. K. Lau is an associate professor in the Department of Industrial and Manufacturing Systems Engineering, The University of Hong Kong. Henry graduated from the University of Oxford with a BA degree in engineering science and a DPhil in Robotics. Prior to joining The University of Hong Kong, he has been working in industry for many years as a systems engineer and section manager at the UK Atomic Energy Authority (UKAEA) and AEA Technology plc., working on projects involving bespoke tele-robotics systems and advanced automation systems for the nuclear industry in decommissioning and waste management. While working in England, Henry was a croucher foundation research fellow at the University of Oxford Robotics Research Group, and a visiting lecturer at Brasenose College teaching Engineering Science. Henry's research interest includes artificial intelligence, in particular in artificial immune systems (AIS), intelligent automation for material handling, virtual and augmented reality systems. 